\title{
A 3D Model of the Human Lung
}

\author{
Tatjana Zrimec ${ }^{1,2}$, Sata Busayarat ${ }^{2}$, and Peter Wilson ${ }^{3}$ \\ ${ }^{1}$ Centre for Health Informatics \\ ${ }^{2}$ School of Computer Science and Engineering \\ University of New South Wales \\ Sydney 2052, Australia \\ \{tatjana, satab\} @ cse.unsw. edu.au \\ ${ }^{3}$ Pittwater Radiology \\ Sydney, Australia
}

\begin{abstract}
This paper presents a method for modelling human lungs using knowledge of lung anatomy and High Resolution CT images. The model consists of a symbolic anatomical structure map and an annotated 3D atlas. The model is implemented using Frame structures. Frames provide a good platform for the comprehensive description of anatomical features and for enabling communication between the image data and the symbolic knowledge. A few important landmarks have been determined and used to divide the lung into clinically meaningful regions, which enable accurate mapping of the model to patient data.
\end{abstract}

\section{Introduction}

A 3D model of the human lung is used in an image understanding system for interpreting HRCT images. The model consists of a qualitative component - a semantic model of lung anatomy and a quantitative component - a 3D atlas. From a set of segmented axial 2D images, a 3D model of the lung is constructed for easy visualization and manipulation (See Fig 1). Relationships among objects in the 3D model are represented using a spatial coordinate system. Our approach differs from the approach proposed in [1] in that we are developing a complete lung model that includes also anatomical information.

\section{Modelling and Representation}

An anatomical model should have the ability to encode the shape of anatomical features and to include normal cases and variations [2]. To assist image processing, a model should include knowledge at both the image level and the anatomical level. Consequently, the knowledge includes descriptions of anatomical components and the relations between them. At the image level, the knowledge includes information about the expected appearance of an anatomical feature in an image. We use a Frame 


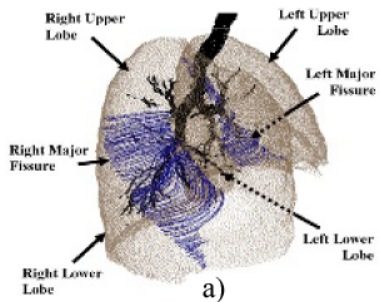

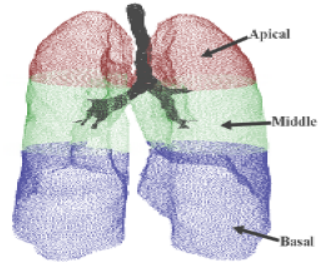

b)

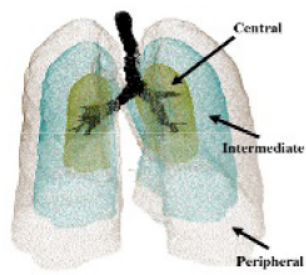

c)

Fig. 1. (a) Lung lobes; Regions: (b) Apex, Medial, Basal; (c) Central, Intermediate, Peripheral.

representation to implement the model, since Frames allow easy representation of the hierarchical structures that are found in the human anatomy [2]. The model includes appearances of the modelled structures from different views. The following Frame types represent the domain knowledge:

Anatomy frame - stores anatomical information about the properties and organization of the lungs and surrounding anatomical structures (for example, spine, lungs, mediastinum).

Structure frame - stores information of the lung structure and lung division: Left lung, Right lung; lung lobes, see Fig. 1. a, and subdivisions down to a particular Bronchopulmonary Segment.

Spatial frame - stores 3D spatial organization and spatial relations of the lung features (apex, hilum, base, borders and surfaces (see Fig. 1. b, c ).

View frame - Volume and $2 D$ - contains a description of a set of axial images as produced by HRCT, and a set of images as produced by X-ray.

Pathology frame - contains knowledge of diseases patters, their expected location and distribution in the lungs.

Currently, we have developed a model of the lung with a 3D lung atlas using a set HRCT volume data from eight cases with 300 to 400 images per study. The lungs were segmented automatically using "Active contour" snakes and morphological operator. Segmented fissures enable division of the lung model into lobes (See Fig. 1. a). A few important landmarks in HRCT images: trachea, sternum, vertebrae, spinal canal, Carina and hilum were segmented automatically and used for lung division into clinically meaningful regions: Apex, Medial, and Base (see Fig. 1. b), Central, Intermediate and Peripheral (see Fig. 1. c). The model facilitates image interpretation and evaluation of the methods for automatic feature segmentation.

\section{References}

1. Li, B., Christensen, E.G., Hoffman, E.A., et.al.: Establishing a Normative Atlas of the Human Lung: Intersubject Warping and Registration of Volumetric CT Images, Acad Radiol, 10 (2003) 255-265

2. Zrimec, T., Sammut, C. “A Medical Image Understanding System”, Engineering applications of Artificial Intelligence 10 (1) (1997) 31-39 